\title{
Atomic-resolution electric field measurements with a universal detector
}

\author{
Jordan A. Hachtel ${ }^{1}$, Arashdeep Thind ${ }^{2}$, Rohan Mishra ${ }^{2,3}$, Juan Carlos Idrobo ${ }^{1}$, Miaofang Chi $^{1}$ \\ ${ }^{1 .}$ Center for Nanophase Materials Science, Oak Ridge National Laboratory, Oak Ridge, TN USA \\ 2. Institute of Materials Science and Engineering, Washington University, St. Louis, MO United States \\ 3. Department of Mechanical Engineering and Materials Science, Washington University, St. Louis, MO \\ United States
}

Differential phase contrast (DPC) imaging is a technique that has yielded significant results in optical, X-ray, and electron microscopy and has demonstrated the ability to image and measure the innate electric field within materials [1]. However, up until recently the maximum spatial resolution achievable by DPC (even in electron microscopy) has been limited to tens of nanometers or more. The incorporation of modern high-speed segmented or pixelated detectors has enhanced the sensitivity of $\mathrm{DPC}$ to a level where atomic-resolution is now possible $[2,3]$.

Here, we demonstrate atomic-resolution DPC imaging utilizing a Hamamatsu ORCA cMOS detector installed on a Nion aberration-corrected UltraSTEM 100. The camera used here is a highly versatile detector that can be installed on most standard microscopes in a straightforward manner, opening up the potential applications of DPC to a wider range of materials and experiments.

By collecting the diffraction plane-projection of the aberration-corrected probe, the Ronchigram, we can collect all the phase contrast required for DPC imaging directly on the cMOS detector. Figure 1a shows a high-angle annular dark field scanning transmission electron microscopy (HAADF-STEM) image of a $\mathrm{CsPbBr}_{3}$ unit cell, an inorganic perovskite with relatively heavy constituent atoms. During the acquisition of the HAADF-STEM image, we simultaneously collect the Ronchigrams corresponding to each probe position on the universal detector. Figures $1 \mathrm{~b}$ and 1c show the Ronchigrams collected from positions on either side of the $\mathrm{Pb}$ site (Fig. 1a); it is observed that on the left side the inherent field of the $\mathrm{Pb}$ atom deflects the electron probe and the intensity on the right side of the Ronchigram is higher, while the inverse is true when the probe is on the right side of the $\mathrm{Pb}$ site.

The effect can be quantified by weighting the intensity in each pixel by how far the pixel position is from the center of the Ronchigram (marked by the dashed line in Fig. 1b and 1c), to form a center-ofmass (COM) image. The weighted COM Ronchigrams for the two pixels are shown in Figures 1d and $1 \mathrm{e}$, and the difference in intensity due to the electric field deflection is more clearly pronounced. By integrating the intensity of all the different pixels in the image, we can calculate the quantity $I_{C O M-X}=$ $\sum X \cdot I_{R}(X)$, where $X$ is the $x$-coordinate of the pixel and $I_{R}(X)$ is the intensity of the Ronchigram on that pixel. The quantity $I_{C O M-X}$ is proportional to the electric field generated by the atom [4]. Thus, by calculating $I_{C O M-X}$ and $I_{C O M-Y}$ for each Ronchigram, we can determine both the intensity and the angle of the electric field with the same spatial-resolution as the as-acquired HAADF image. Figure 1f shows the same HAADF-STEM image shown in Fig. 1a with arrows indicating the intensity and direction of the electric field at the atomic scale across the $\mathrm{CsPBBr}_{3}$ unit cell.

Beyond demonstrating atomic-resolution DPC acquired with a universal detector, we will further establish the extent of DPC spatial resolution by using the technique to examine light atoms in a distorted perovskites and directly examine electric fields at atomically-sharp interfaces and boundaries. 


\section{References:}

[1] N.H. Dekkers \& H. de Lang. Optik. 41 (1974), p. 452.

[2] N. Shibata et al., Nat. Phys. 8 (2012), p. 611.

[3] H. Yang et al., Nat. Comm. 7 (2016), ncomms12532

[4] R. Close et al., Ultramicroscopy 159 (2015), p. 124

[5] Research supported by ORNL's Center for Nanophase Materials Sciences, which is a U.S.

Department of Energy Office of Science User Facility

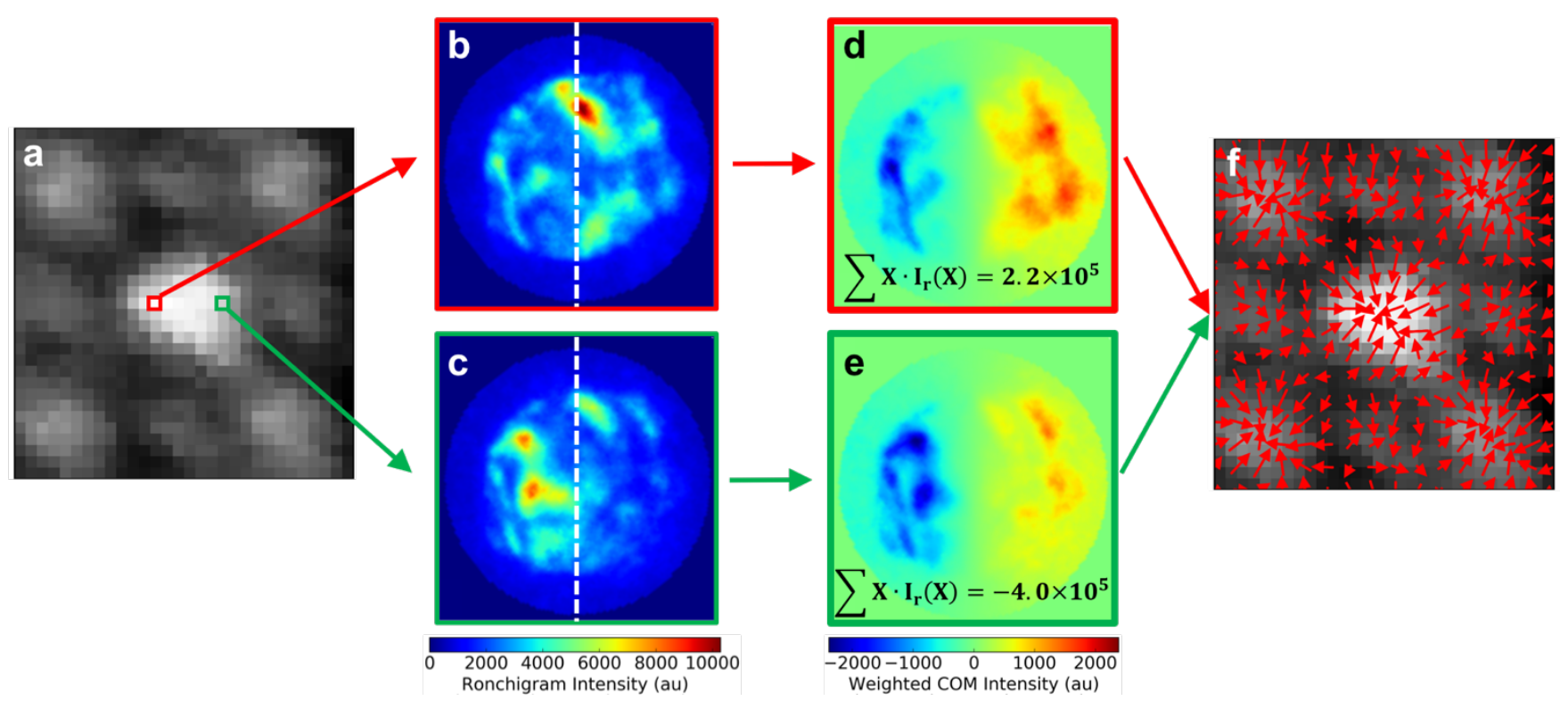

Figure 1. (a) HAADF-STEM image of $\mathrm{CsPbBr}_{3}$ perovskite unit cell. (b,c) Simultaneously acquired Ronchigram images from the pixels marked on the left (red - b) and right (green - c) of $\mathrm{Pb}-\mathrm{Br}$ atomic column; when probe is on the left side of $\mathrm{Pb}$ atom, Ronchigram intensity is higher on the right side; vice versa when the probe is on right side of $\mathrm{Pb}$ atom. (d,e) COM Ronchigrams are generated by weighting each pixel in the regular Ronchigram by $x$-distance from Ronchigram center (marked with the dashed line in (b) and (c)), allowing for calculating $\mathrm{I}_{\mathrm{COM}-\mathrm{X}}$ value of Ronchigram, which is proportional to $x$ component of electric field. (f) Calculating $\mathrm{I}_{\mathrm{COM}-\mathrm{X}}$ and $\mathrm{I}_{\mathrm{COM}-\mathrm{Y}}$ values for every pixel in unit cell can be used to plot magnitude and direction of electric field at atomic scale. 